\title{
Calibration and Use of Intraoperative Cone-Beam Computed Tomography: An In-Vitro Study for Wrist Fracture
}

\author{
Erin Janine Smith ${ }^{1}$, Anton Oentoro ${ }^{2}$, Hisham Al-Sanawi ${ }^{3}$, Braden Gammon ${ }^{3}$, \\ Paul St. John ${ }^{4}$, David R. Pichora ${ }^{3,4}$, and Randy E. Ellis ${ }^{1,2,3,4}$ \\ ${ }^{1}$ Mechanical Engineering, Queens University at Kingston, Canada K7L 3N6 \\ 2 School of Computing, Queen's University, Kingston Ontario, Canada K7L 3N6 \\ ${ }^{3}$ Department of Surgery, Kingston General Hospital, Kingston, Canada K7L 2V7 \\ ${ }^{4}$ Human Mobility Research Centre, Queen's University, Kingston, Canada K7L 2V7 \\ ellis@cs.queensu.ca
}

\begin{abstract}
The standard workflow in many image-guided procedures, preoperative imaging followed by intraoperative registration, can be a challenging process and is not readily adaptable to certain anatomical regions such as the wrist. In this study we present an alternative, consisting of a preoperative registration calibration and intraoperative navigation using 3D cone-beam CT. A custom calibration tool was developed to preoperatively register an optical tracking system to the imaging space of a digital angiographic $\mathrm{C}$-arm. This preoperative registration was then applied to perform direct navigation using intraoperatively acquired images for the purposes of an in-vitro wrist fixation procedure. A validation study was performed to assess the stability of the registration and found that the mean registration error was approximately $0.3 \mathrm{~mm}$. When compared to two conventional techniques, our navigated wrist repair achieved equal or better screw placement, with fewer drilling attempts and no additional radiation exposure to the patient. These studies suggest that preoperative registration coupled with direct navigation using procedurespecific graphical rendering, is potentially a highly accurate and effective means of performing image-guided interventions.
\end{abstract}

\section{Introduction}

Minimally invasive computer-assisted interventions in orthopedics have, to date, largely relied on preoperative computed tomography (CT) scans. In the usual paradigm, the images are segmented, a plan is made, intraoperative registration of the image to the patient is performed, and only then can the surgeon navigate the procedure. There are many drawbacks to this paradigm, including: logistical challenges associated with preoperative CT; time-consuming segmentation, with skilled technicians often requiring an hour or more; attachment of a tracking device to the anatomy can be difficult or impossible; and achieving the registration is invasive, time-consuming, and fraught with errors.

Recent developments in flat-panel fluoroscopy have made 3D cone-beam CT (CBCT) feasible for intraoperative use in an operating room. Such 3D

T. Jiang et al. (Eds.): MICCAI 2010, Part III, LNCS 6363, pp. 359-366, 2010.

(C) Springer-Verlag Berlin Heidelberg 2010 
volumetric data can be used, with direct navigation, in a system that requires neither image segmentation nor registration during the surgical procedure. Instead, a direct-navigation system can use a preoperative calibration stage to register the coordinate frame of the image space to the coordinate frame of a fixed tracking system. The resulting workflow needs only a minimally invasive tracking device attached to a patient, with no segmentation or surface-based registration - nearly ideal for computer-assisted minimally invasive procedures.

Direct navigation is especially attractive for wrist fractures, particularly for the scaphoid, one of the eight carpal bones that is frequently fractured following a fall on an outstretched palm [1. Proximal scaphoid fractures often fail to unite, due to the unique blood supply to this region. As such, primary internal fixation is often a preferred method of treatment, and typically involves the placement of a Kirschner wire (K-wire) or surgical screw along the central axis of the bone to unite the bony fragments.

Clinical studies have indicated the importance of an accurate placement along the central axis for a successful outcome [2, with minimally invasive percutaneous technique preferred to reduce the risk of infection and compromising nearby tissue structures [3. However, percutaneous pinning is challenging because of the small size of the scaphoid, and requires many intra-operative X-ray images to ensure an accurate placement of the fixation device. As fluoroscopic images are $2 \mathrm{D}$, it is also difficult to correctly interpret the three-dimensional anatomy in order to locate the central axis of the scaphoid.

It is difficult to adapt the traditional computer-assisted surgical workflow to navigate scaphoid pinning because a surface-based or fiducial-based registration cannot be used successfully in the region of the wrist 4 . Attempts at imagebased registration using fluoroscopic images to match extracted contours [5] or using the data directly with mutual information [6] have so far been insufficiently accurate for clinical use. Ultrasound techniques [4 have not been shown to improve the application accuracy over conventional fluoroscopy.

Here, we present a novel technique for preoperative calibration and results of a preliminary validation assessment. We then apply our preoperative calibration to perform intraoperative image guidance for volar percutaneous scaphoid pinning, and compare this technique to conventional fluoroscopic guidance using a standard C-arm and guidance using a digital angiographic C-arm.

\section{Preoperative Calibration: Materials and Methods}

Our direct navigation solved the image-to-patient registration problem by splitting the chain of coordinate transformations into two pieces, preoperative and intraoperative. Preoperatively, the pose of the 3D CBCT was found in tracking coordinates by using a custom calibration device; later, the pose of the patient was captured simultaneously with the CBCT image acquisition. The key technical question was the accuracy and reliability of the preoperative calibration.

All experiments were performed in a recently-constructed operating room at Kingston General Hospital. This room was integrated with an Optotrak Certus 
(Northern Digital Inc., Waterloo, Canada) tracking system and a flat-panel 3D fluoroscope (Innova 4100, GE Healthcare, Buc, France) that acquired CBCT images. A custom surgical navigation system was used to provide image guidance.

For full details of the preoperative calibration process, the reader is referred to a recent Master's thesis [7. A custom Multi-Modal Calibrator (MMC) was developed to conduct a preoperative calibration in order to relate the coordinate frames of the Optotrak $\{\mathbf{C}\}$ and that of the Innova 3D spin image $\{\mathbf{S}\}$. The device was shaped like a hollow cube (shown in Fig. 1). To be sensed by both the imaging device and the position sensor of the Optotrak, the MMC contained respectively metallic radio-opaque markers and infrared light-emitting diodes.

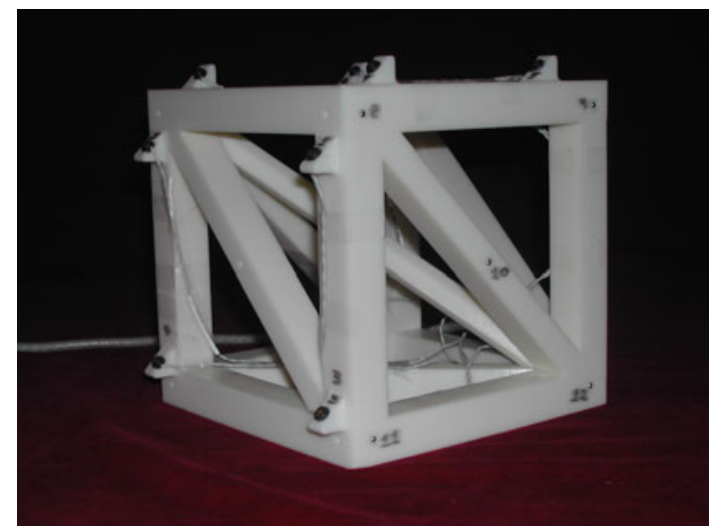

Fig. 1. A photograph of the multi-modal calibration device that was used for this study

Prior to a navigated surgical session, the registration was performed by positioning the $\mathrm{MMC}$ on the operating table. The CBCT image was acquired and, simultaneously, the pose ${ }_{M}^{C} T$ was acquired to find the MMC in Optotrak Certus coordinates $\{\mathbf{C}\}$. The MMC beads were found in the CBCT image using a validated interpolation algorithm $[8$; because the algorithm also detected wires and IREDs, a variant of the robust RANSAC algorithm [9] was used to register the detected beads, yielding the transformation ${ }_{M}^{S} T$. This gives the critical calibration equation

$$
{ }_{S}^{C} T={ }_{M}^{C} T *{ }_{S}^{M} T
$$

that coupled any subsequent CBCT image to Optotrak coordinates.

The precalibrated registration was studied in an extensive experiment over 3 weeks. During this time, the MMC was left undisturbed on the operating table and $14 \mathrm{CBCT}$ scans were acquired periodically. The analysis of the transformations used the differential transform $\Delta T$ between transforms $T_{A}$ and $T_{B}$

$$
\Delta T=T_{B} * T_{A}^{-1}
$$

Two sets of comparisons were made: pairwise ${ }_{M}^{C} T$ using $\Delta^{C} T_{i}$, and pairwise ${ }_{S}^{M} T_{i}$ using $\Delta^{S} T_{i}$. The rotational component of each $\Delta T$ was converted to an angle/axis notation, and for the translational component the norm was calculated. 


\section{Preoperative Calibration: Results}

The 14 analyses of the transforms ${ }_{M}^{C} T_{i}$ and ${ }_{S}^{M} T_{i}$ were examined statistically. The mean rotational and translational deviations in the dimensions of the operating room are presented in Table 1. The mean rotational and translational deviations in the movement of the Innova system are presented in Table 2.

Table 1. Average rotational and translational errors calculated from $\Delta^{C} T$ for the Innova registration experiment. $\boldsymbol{\mu}$ is the mean and $\boldsymbol{\sigma}$ is the standard deviation.

\begin{tabular}{|r|r|r|r|}
\hline Type of Error & $\boldsymbol{\mu}$ & $\boldsymbol{\sigma}$ & Range \\
\hline Rotational Error(degrees) & - & - & - \\
\hline Translational Error for X $(\mathrm{mm})$ & 0.19 & 0.059 & $0.0035-0.44$ \\
\hline Translational Error for Y $(\mathrm{mm})$ & 0.059 & 0.0062 & $0.00010-0.18$ \\
\hline Translational Error for Z $(\mathrm{mm})$ & 0.19 & 0.029 & $0.00070-0.67$ \\
\hline Norm of Translational Error $(\mathrm{mm})$ & 0.30 & 0.176 & $0.028-0.74$ \\
\hline
\end{tabular}

Table 2. Average rotational and translational errors calculated from $\Delta^{S} T$ for the Innova registration experiment; $\boldsymbol{\mu}$ is the mean and $\boldsymbol{\sigma}$ is the standard deviation

\begin{tabular}{|r|r|r|r|}
\hline Component & $\boldsymbol{\mu}$ & $\boldsymbol{\sigma}$ & Range \\
\hline Rotational Error(degrees) & 0.27 & 0.027 & $0.0065-0.61$ \\
\hline Translational Error for X (mm) & 0.036 & 0.0040 & $0.00030-0.12$ \\
\hline Translational Error for Y $(\mathrm{mm})$ & 0.053 & 0.0080 & $0.0016-0.14$ \\
\hline Translational Error for Z (mm) & 0.058 & 0.0083 & $0.00050-0.15$ \\
\hline Norm of Translational Error $(\mathrm{mm})$ & 0.097 & 0.0076 & $0.020-0.17$ \\
\hline
\end{tabular}

The mean registration error was approximately $0.3 \mathrm{~mm}$, or 300 microns. This compares favorably with the stated accuracy of the Optotrak (150 microns) and is unlikely to be improved upon using our equipment. We concluded that preoperative calibration is a fast and highly accurate method for direct navigation.

\section{In-Vitro Application: Materials and Methods}

Direct navigation was applied to a volar percutaneous pinning procedure. This technique was compared to two conventional imaging techniques: (i) "C-arm", in which a standard fluoroscopic C-arm was used, and (ii) "Innova", in which the Innova imager was programmed with orthogonal $2 \mathrm{D}$ views.

A 3D model of the human wrist was constructed to provide greater consistency between the trials than permissible using cadaveric anatomy (Fig. 2). The model wrist featured an interchangeable scaphoid which allowed the wrist to be reused for each trial by replacing only the scaphoid. A molded recess was integrated into the model which served to firmly seat the scaphoid, and to which the scaphoid was secured using a plastic fastener. 


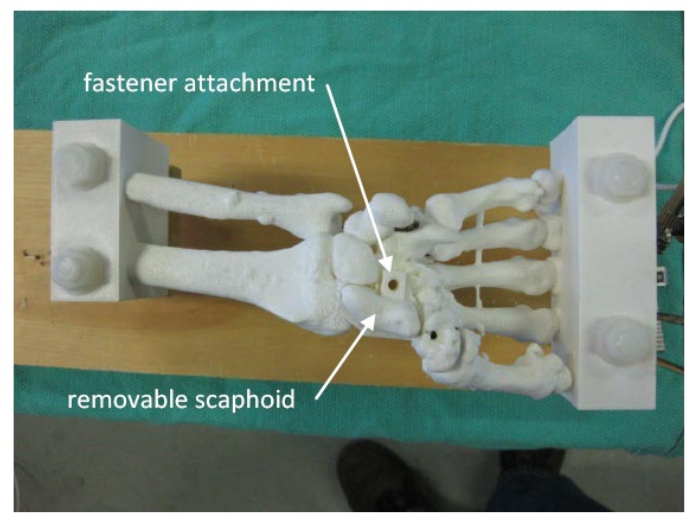

(a)

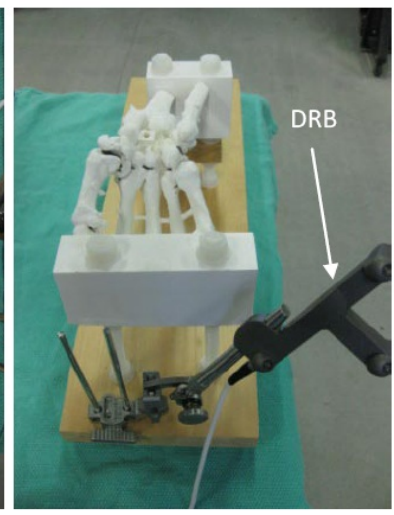

(b)

Fig. 2. 3D Wrist Model. (a) A removable scaphoid was seated in a molded recess and secured via a fastener hole. (b) The navigated trial setup with a patient coordinate frame $\mathbf{P}$ established by an optical tracking array(DRB).

Both the model wrist and removable scaphoids were fabricated using a 3D rapid prototyping printer (SST 1200es, Dimension/Stratasys, Eden Prairie, USA). To facilitate drilling, the scaphoid models were printed with a medium density polymer then coated with barium-infused paint. The wrist was printed with lower density polymer. During each trial, the entire apparatus was covered to prevent direct visualization of the bony anatomy but to permit realistic palpation.

Randomized trials were conducted in which three surgeons each performed 24 trials -8 using each imaging technique. The surgical goal was to insert a $1.6 \mathrm{~mm} \mathrm{~K}$-wire along the central axis of the model scaphoid. In each technique a volar approach was employed in which the wire was drilled from the distal end until it breached the proximal end. This exit hole was created to allow for post-trial data analysis. For each trial, the number of drill passes was recorded. To assess radiation exposure, an exposure meter was positioned adjacent to the wrist model to estimate a patient's radiation dose.

An optical tracking array was attached at the base of the plastic model to establish a patient coordinate frame $\{\mathbf{P}\}$. An intraoperative image of the wrist was captured using the Innova to perform a $3 \mathrm{D}$ acquisition and, simultaneously, the navigation system captured the pose of $\{\mathbf{P}\}$ in the Optotrak coordinate frame $\{\mathbf{C}\}$ as the transformation ${ }_{C}^{P} T$. The combined registration transformation was the composition of the preoperative calibration transform ${ }_{S}^{C} T$ (Eq. 1) with the transformation ${ }_{C}^{P} T$ to find the overall image-to-patient registration as:

$$
{ }_{S}^{P} T={ }_{C}^{P} T *_{S}^{C} T
$$

The navigation system provided digitally reconstructed radiographs to the surgeon (Fig. 3). In the planning phase, the surgeon positioned a desired drill path on the rendered views. During the drilling phase, the real-time orientation of a tracked drill guide relative to this planned path was shown on the overhead OR 


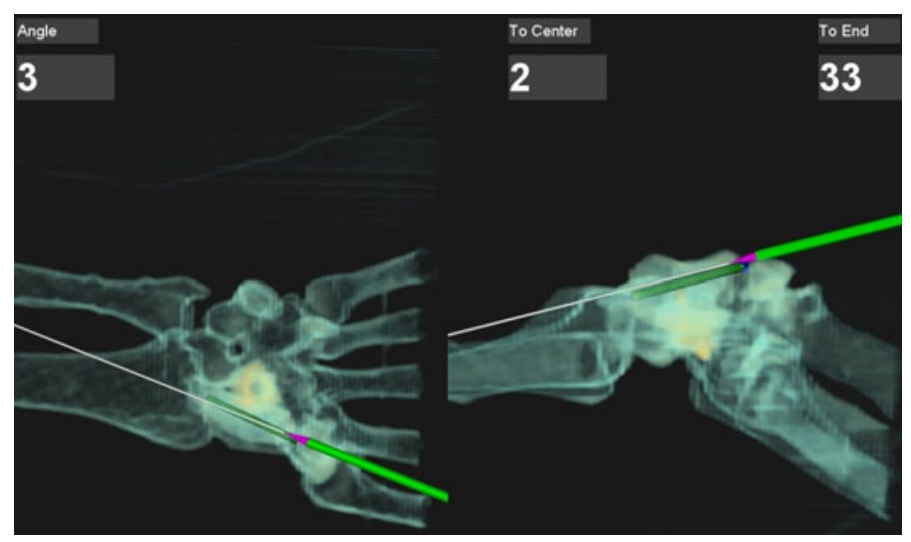

Fig. 3. Screen shot of the software during a navigated procedure showing orthogonal views. The dark green cylinder depicts the target plan; the purple and green stylus represents the real-time tracked drill guide.

monitors. In conventional techniques, surgeons were given the option of using an identical untracked drill guide.

Following the trials, CT scans of the drilled scaphoids were obtained and segmented (Mimics v13, Materialise, Leuven, Belgium) in order to generate 3D surface models for data analysis. Algorithms were developed to determine the line of best fit through the drill hole and compute the shortest distance from this line to the exterior surface of the scaphoid. This measure was extrapolated to determine the location of the shortest distance and to assess whether a placed screw would have breached the scaphoid. Two-sample F-tests were used to assess equal variances and select appropriate t-tests for wire placement centricity, drill passes, and radiation exposure.

\section{In-Vitro Application: Results}

The 24 scaphoid drilling trials were analyzed to assess screw placement, number of drill passes required, and x-ray exposure to the patient(Table 3). The minimum distance from the drill path to the scaphoid surface was deemed to be credible a measure of centricity as well as a safety factor for screw breach. Although no potential screw breaches were noted in any of the trials, the minimum distance from the drill path to the scaphoid surface was significantly higher using navigation when compared to the Innova $(\mathrm{p}=0.02, \alpha=0.05)$.

There was no significant difference in the number of drill passes between the navigated and Innova techniques, however the $\mathrm{C}$-arm technique required more attempts $(\mathrm{p}=0.01, \alpha=0.05)$. Imager output was significantly higher in the navigated cases (mean $\left.=11.05 \mathrm{cGy} / \mathrm{m}^{2}, \mathrm{SD}=1.67 \mathrm{cGy} / \mathrm{m}^{2}\right)$ than with the Innova $\left(\right.$ mean $\left.=2.78 \mathrm{cGy} / \mathrm{m}^{2}, \mathrm{SD}=2.13 \mathrm{cGy} / \mathrm{m}^{2}\right)$ or C-arm $($ mean $=$ $\left.0.03 \mathrm{cGy} / \mathrm{m}^{2}, \mathrm{SD}=0.03 \mathrm{cGy} / \mathrm{m}^{2}\right)$. Conversely, there was no significant difference 
Table 3. Summary of post-trial analysis of the three drilling methods: Navigated (N), Innova (I) and C-arm (C)

\begin{tabular}{|r|c|c|c|}
\hline & N & I & C \\
\hline Safety [mm] & $2.8 \pm 0.5$ & $2.6 \pm 0.5$ & $2.8 \pm 0.5$ \\
\hline X-ray Exposure[mrad] & $1.1 \pm 1.4$ & $0.5 \pm 0.9$ & $2.3 \pm 3.3$ \\
\hline Drill Passes & $1.5 \pm 0.7$ & $1.7 \pm 1.0$ & $2.2 \pm 0.8$ \\
\hline
\end{tabular}

in the mean imaging time. More importantly, no difference in patient x-ray exposure (as recorded by the exposure meter) was observed.

\section{Discussion}

An important consideration in our workflow was the overall speed of the preoperative registration step. The entire registration process, including scanning, transfer, and computations, took less than 15 minutes to perform. In an ideal scenario, where the relative position and orientation of the imaging modalities and the position sensor remain perfectly fixed, the registration process would need to be performed only once. This is unlikely in a realistic clinical setting, so the registration process is expected to be performed more often - perhaps daily - and thus needs to be reasonable efficient.

The results from our preliminary trial demonstrate that we can achieve equal or better safety of screw placement, with fewer drill passes and, no additional radiation exposure to the patient. Of a potentially greater concern is the radiation exposure to the surgeon, which we are currently in the process of evaluating in a secondary round of trials, through the use of radiation badges. It is worth noting that we expect the radiation exposure to the surgeon to be minimal, as the initial 3D acquisition spin is acquired while the surgeon is outside of the radiation field, and the only notable exposure would be the potential use of supplementary fluoroscopic images to verify screw placement.

One of the main limitations of our current navigation system is that it only provides the orientation of the drill guide and does not provide any depth cues. Feedback from the surgeons indicated that this was indeed an impediment, and forced them to rely on using more intraoperative 2D fluoroscopic images to verify wire position. Amending the navigation to include depth cues is now a focus for upcoming trials. Another area of improvement for the next version of the navigation system concerns the techniques used to render the 3D image. Again, surgeons have expressed difficulty with viewing the margins of the scaphoid on the rendered views, and thus planning the procedure. We are currently experimenting with new rendering and visualization techniques for upcoming trials.

\section{Conclusion}

A novel workflow for image-guided surgery was presented that does not require conventional intraoperative image registration. The system has a much simpler 
and efficient workflow than conventional CT image acquisition and segmentation without compromising accuracy. We have also demonstrated that this technique can be adapted to anatomical regions, such as the wrist, that cannot be readily registered and spatially tracked intraoperatively. Our future challenges will be to make this system practical for routine clinical use.

Acknowledgements. This work was supported in part by the Canada Foundation for Innovation, the Canadian Institutes for Health Research, Kingston General Hospital, the Ontario Innovation Trust, and the Natural Sciences and Engineering Research Council of Canada.

\section{References}

1. Leslie, I., Dickson, R.: The fractured carpal scaphoid. J. Bone Joint Surg. Br. 63-B, 225-230 (1981)

2. McCallister, W., Knight, J., Kaliappan, R., Trumble, T.: Central placement of the screw in simulated fractures of the scaphoid waist. J. Bone Joint Surg. Am. 85-A, 72-77 (2003)

3. Filan, S., Herbert, T.: Herbert screw fixation of scaphoid fractures. J. Bone Joint Surg. Br. 78-B, 519-529 (1996)

4. Beek, M., Abolmaesumi, P., Luenam, S., Ellis, R., Sellens, R., Pichora, D.: Validation of a new surgical procedure for percutaneous scaphoid fixation using intraoperative ultrasound. Medical Image Analysis 12, 152-162 (2008)

5. Feldmar, J., Ayache, N., Betting, F.: 3D-2D projective registration of free-form curves and surfaces. Comput. Vis. Image Underst. 65(3), 403-424 (1997)

6. Dalvi, R., Abugharbieh, R., Pickering, M., Scarvell, J., Smith, P.: Registration of $2 \mathrm{D}$ to $3 \mathrm{D}$ joint images using phase-based mutual information. Proc. Soc. Photo Opt. Instrum. Eng. 651209, 1-9 (2007)

7. Oeontoro, A.: A system for computer-assisted surgery with intraoperative ct imaging. Master's thesis, Queen's University, Kingston, Canada (2009)

8. Ellis, R.E., Toksvig-Larsen, S., Marcacci, M., Caramella, D., Fadda, M.: Use of a biocompatible fiducial marker in evaluating the accuracy of CT image registration. Investigative Radiology 31(10), 658-667 (1996)

9. Fischler, M.A., Bolles, R.C.: Random sample consensus: A paradigm for model fitting with applications to image analysis and automated cartography. Communications of the ACM 24(3), 381-395 (1981) 